\title{
Studentized Extreme Eigenvalue Based Double Threshold Spectrum Sensing Under Noise Uncertainty
}

\author{
Cebrail ÇiFLIKLI, FatihYavuz ILGIN
}

\begin{abstract}
The eigenvalue based spectrum sensing is a low-cost spectrum sensing method that detects the presence of the licensed user signal in desired frequency. Traditional single-threshold eigenvalue sensing methods, which are widely used in the literature, can exhibit inadequate performance under low SNR and noise uncertainty. Therefore, in this study an eigenvalue-based spectrum sensing method is proposed using a double threshold with the studentized extreme eigenvalue distribution function The results that threshold values obtained for the proposed method were simulated in Rayleigh fading channels. The results were compared with traditional methods and they were observed to be more accurate.
\end{abstract}

Keywords: cognitive radio; covariance matrix; noise uncertainty; spectrum sensing; Tracy-Widom distribution

\section{INTRODUCTION}

The studies done by the Federal Communications Commission (FCC) revealed that the spectrum has not been used enough due to traditional fixed spectrum allocation policies [1, 2]. For this reason, in Cognitive Radio (CR) systems, to increase spectrum efficiency the secondary users borrow the remaining spectrum from the primary users. CR has the ability to detect a wide spectrum of the environment that it is in or a specific part of the spectrum $[3,4]$. For that reason a successful spectrum sensing is the most important start stage for cognitive radio systems. For executing prospering spectrum sensing performance, many methods based on single threshold methods user are proposed in literature $[5,6]$. Due to multi-path fading, noise uncertainty and low SNR, single-threshold methods can perform very poorly [7-13]. To overcome these problems and to increase the probability of detection even at low SNR values, double-threshold spectrum sensing methods have been proposed [13-16]. Among these methods, energy detection has attracted attention for years because of its low calculation cost. The eigenvalue sensing methods are generally preferred, in as much as they do not require any prior knowledge (variance, modulation, channel knowledge) about the noise signal and the primary user signal while using the statistical properties of the signal and noise. Therefore, expressing the statistical properties correctly is quite effective in the performance of the method.

In this study, we use a new eigenvalue distribution function for the dual-threshold eigenvalue based spectrum sensing method. For the proposed method, thresholds equations were obtained, the theoretical analyses were simulated in Rayleigh fading channel and their results were interpreted.

The remainder of this paper is organized as follows. In Section 2, on the double threshold spectrum sensing model with studentized extreme eigenvalue distribution is presented. An eigenvalue-based spectrum sensing method using a double threshold with the studentized extreme eigenvalue distribution is proposed in Section 3. In Section 4, simulation results are presented. Finally, in Section 5, some concluding remarks are made.
In this paper, while the bold letters represent the matrix and the normal letters represent the vectors, $(\cdot)^{\prime}$, var $(\cdot)$ and $\mathrm{E}(\cdot)$ indicates transpose, variance and the mathematical expectation respectively.

\section{DOUBLE THRESHOLD SPECTRUM SENSING MODEL}

In general terms, spectrum sensing is to determine whether a primary user signal is present in a specific spectrum region that has a limited bandwidth and center frequency $f_{\mathrm{c}}$. This process can be formulated mathematically in the following way, by a binary hypothesis described as follows:

$$
\begin{aligned}
& H_{0}: \boldsymbol{x}(k)=\boldsymbol{\eta}(k) \\
& H_{1}: \boldsymbol{x}(k)=\boldsymbol{s}(k)+\boldsymbol{\eta}(k)
\end{aligned}
$$

Where $\boldsymbol{x}(k)$ represents a specific spectrum region. $\boldsymbol{s}(k)$ denotes the primary user's signal, and $\boldsymbol{\eta}(k)$ shows zeromean, independent and identically distributed (i. i. d.) white Gaussian noise. $H_{0}$ represents the absence of the primary user mark on the related frequency or the related frequency band is vacant. The $H_{1}$ hypothesis indicates that primary user signal is present in the current frequency or that the related frequency band is busy.

In traditional spectrum sensing, secondary users decide only if the spectrum is occupied / vacant by looking at a threshold value. In the dual-threshold method, however, there are two thresholds for making this decision. In the relevant method, there are two thresholds as $\gamma_{l o}$ and $\gamma_{u p}$ thresholds to measure the reliability of the decision made. As a result of comparing the test statistic (TS) value with the threshold values, it is decided that the spectrum is occupied/vacant. The "confusion region" means that observation is not sufficiently reliable. The aim here is to keep the probability of detection above a certain value even at the lowest SNR. The traditional one-threshold method and the dual-threshold method are shown in Fig. 1 respectively.

Where $\gamma, \gamma_{l o}$ and $\gamma_{u p}$ denotes threshold in singlethreshold systems, lower threshold in double-threshold systems and upper threshold in double-threshold systems respectively. Thereby, $T S>\gamma_{u p}$ is the primary user in the 
spectrum and $T S>\gamma_{l o}$ is the vacant spectrum. However, if $\gamma_{u p}<T S<\gamma_{u p}$ is present, the spectrum needs to be reevaluated. This is expressed mathematically, in the following way.

$$
=\left\{\begin{array}{l}
\text { decision } \\
\text { spectrum vacant } T S>\gamma_{u p} \\
\text { resense spectrum } \gamma_{u p}<T S>\gamma_{u p} \\
\text { spectrum occupied } T S>\gamma_{l o}
\end{array}\right.
$$



(a)

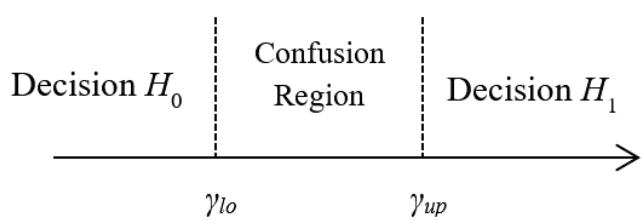

(b)

Figure 1 Conventional detection method with one threshold (a) Hierarchical spectrum sensing method with double-thresholds method (b)

\section{PROPOSED METHOD}

In the proposed method, we consider the problem of spectrum sensing with the signals which are detected by a secondary user with $m$ antennas through Rayleigh fading channels. We can express the hypotheses $H_{0}$ and $H_{1}$ mathematically as follows $[9,11]$.

$\boldsymbol{x}(k)=\Gamma \boldsymbol{h}(k) \boldsymbol{s}(k)+\boldsymbol{\eta}(k), k=1,2, \ldots, n$

Where $\Gamma=0$ under $H_{0}, \Gamma=1$ under $H_{1}$ and $\boldsymbol{x}(k)$ denotes the signal that the secondary user detects with $m$ antennas. $\boldsymbol{\eta}(k)=\left[\eta_{1}(k), \eta_{2}(k), \ldots, \eta_{m}(k)\right]^{\mathrm{T}}$ represents the independent and identically distributed circularly symmetric complex Gaussian (CSCG) white noise with mean zero and covariance $\sigma_{n}^{2} \boldsymbol{I}_{\boldsymbol{m}}, n(k) \sim C N\left(0, \sigma_{n}^{2} \boldsymbol{I}_{\boldsymbol{m}}\right)$ with $\sigma_{n}^{2}$ being the noise power. $s(k)$ is the deterministic but its instantaneous power unknown primary user signal and $\boldsymbol{h}(k)$ is the Rayleigh fading channel in the way that $\boldsymbol{h}(k)=\left[h_{1}(k)\right.$, $\left.h_{2}(k), \ldots, h_{m}(k)\right]^{\mathrm{T}}$. Thus, the statistical covariance matrixes of the received signal for $H_{0}$ and $H_{1}$ states, are defined as follows.

$$
\begin{aligned}
& H_{0} \rightarrow \boldsymbol{R}_{\boldsymbol{x}} \cong \frac{1}{n} \sum_{k=1}^{n} \boldsymbol{x}(n) \boldsymbol{x}^{\prime}(n)=\left(\begin{array}{ccc}
r_{11} & \cdots & r_{1 m} \\
\vdots & \ddots & \vdots \\
r_{m 1} & \cdots & r_{m n}
\end{array}\right) \\
& H_{1} \rightarrow \boldsymbol{R}_{\boldsymbol{\eta}} \cong \frac{1}{n} \sum_{k=1}^{n} \boldsymbol{\eta}(n) \boldsymbol{\eta}^{\prime}(n)=\left(\begin{array}{ccc}
r_{11} & \cdots & r_{1 m} \\
\vdots & \ddots & \vdots \\
r_{m 1} & \cdots & r_{m n}
\end{array}\right)
\end{aligned}
$$

In this case, let $\lambda_{1}<\lambda_{2}<, \ldots,<\lambda_{p}$ and $\rho_{1}<\rho_{2}<, \ldots$, $<\rho_{p}$ denote the eigenvalues of $\boldsymbol{R}_{\boldsymbol{\eta}}$ and $\boldsymbol{R}_{\boldsymbol{x}}$, respectively, where $\boldsymbol{R}_{\boldsymbol{\eta}}$ is almost a Wishart random matrix. Spectral decomposition of a random matrix has been studied in mathematics and statistics in recent years, while studies that define the probability density function of the largest eigenvalue of a Wishart random matrix as a closed function have been done [14].

Theorem 1. Assuming that the noise is complex let

$$
\begin{aligned}
& A(n)=\frac{n}{\sigma_{\eta}^{2}} \boldsymbol{R}_{\boldsymbol{\eta}}, \quad \mu_{n, m}=n^{-1}(\sqrt{n-1 / 2}+\sqrt{m-1 / 2})^{2}, \\
& \sigma_{n, m}=\left(\mu_{n, m} / n\right)^{1 / 2}\left(\frac{1}{\sqrt{n-1 / 2}}+\frac{1}{\sqrt{m-1 / 2}}\right)^{1 / 3} \text { and } \\
& \mu_{n, m, 0}=\mu_{n, m}+a \sigma_{n, m, 0} \text { is } \sigma_{c}^{2}=\frac{n m}{2+n m}\left(\sigma_{n, m, 0}^{2}-\mu_{n, m, 0}^{2}\right),
\end{aligned}
$$

where $b$ and $\sigma_{n, m, 0}=b \sigma_{n, m}$ respectively. Therefore $\frac{\lambda_{\max } A(n)-\mu}{v}$ converge to the Tracy-Widom distribution of order 1 [17].

Theorem 2. Assuming that $\frac{n}{m}=y$ and $0<y<1$, then, $\lambda_{\min }=\sigma_{\eta}^{2}(1-\sqrt{n})^{2}$. Based on these theorems, when $n$ is adequately large, the center values of the largest and smallest eigenvalues are $\frac{\sigma_{\eta}^{2}}{n}(\sqrt{n}-\sqrt{m})^{2}$ and $\frac{\sigma_{\eta}^{2}}{n}(\sqrt{n}+\sqrt{m})^{2}$, respectively [9].

The Tracy-Widom distributions were found by Tracy and Widom. This theorem defines the largest eigenvalue distribution of the covariance matrix of the random hermit matrix. Let $F_{1}$ be the cumulative distribution function (CDF) of the Tracy-Widom distribution of order 1. This distribution function is defined as follows:

$$
F_{1}(t)=\exp \left(\int_{t}^{\infty}\left(q(u)+(u-t) q^{2}(u)\right) \mathrm{d} u\right)
$$

where $q(u)$ is the solution of the nonlinear Painleye II differential equation and is defined as follows,

$$
q^{\prime \prime}(u)=u q(u)+2 q^{3}(u)
$$

The specific values for this function are given in Tab. $1[9,10]$

Table 1 Some numerical values for the Tracy-Widom distribution of order 1

\begin{tabular}{|c|c|c|c|c|c|}
\hline$x$ & $-3,90$ & $-2,78$ & $-1,27$ & 0,45 & 2,02 \\
\hline$F_{1}(x)$ & 0,01 & 0,10 & 0,50 & 0,90 & 0,99 \\
\hline
\end{tabular}

\subsection{Derivation of Upper Threshold Value $\left(\gamma_{u p}\right)$}

According to the detection theory, the equations of probability of false alarm $\left(P_{f a}\right)$ and probability of detection $\left(P_{m}\right)$ are defined by conditional probability conditions that are $P\left(T S>\gamma_{u p} \mid H_{0}\right)$ and, $P\left(T S<\gamma_{l o} \mid H_{1}\right)$ respectively. Where, TS, $\gamma_{l o}, \gamma_{u p}$, denote the test statistic and high threshold and low threshold, respectively (Fig. 1). The high 
threshold value is calculated in a way similar to the singlethreshold method. $\gamma_{u p}$, can be calculated by the following equation:

$P_{f a}=P\left(H_{1} \mid H_{0}\right)=P\left(\frac{\lambda_{\max }\left(\boldsymbol{R}_{\boldsymbol{\eta}}\right)}{\lambda_{\min }\left(\boldsymbol{R}_{\boldsymbol{\eta}}\right)}>\gamma_{u p}\right)$

where, to obtain the threshold value one side of the equation must be imitated to the Tracy-Widom distribution of order 1 . To this end $\mu_{n, m, 0}$ we $\sigma_{c}$ values should be added to both sides of the equation.

$$
\begin{aligned}
& P_{f a}=P\left(\lambda_{\max }\left(\boldsymbol{R}_{\boldsymbol{\eta}}\right) \frac{n}{\sigma_{\eta}^{2}} \frac{\sigma_{\eta}^{2}}{n}>\gamma_{u p} \lambda_{\min }\left(\boldsymbol{R}_{\boldsymbol{\eta}}\right)\right) \\
& P_{f a}=P\left(\left(\frac{\lambda_{\max }(A(n))-\mu_{n, m, 0}}{\sigma_{c}}\right)>\gamma_{u p}\left(\frac{(\sqrt{n-\sqrt{m}})^{2}-\mu_{n, m, 0}}{\sigma_{c}}\right)\right)
\end{aligned}
$$

where $A(n)$ denotes normalization coefficient. Finally, using the survival equation, $\gamma_{u p}$, threshold is calculated as follows [12]:

$$
\gamma_{u p}=F_{1}^{-1}\left(\frac{\sigma_{c}^{2}}{1-P_{f a}}\right)\left(\frac{\sqrt{\frac{n m}{2+n m}\left(\sigma_{n, m, 0}^{2}-\mu_{n, m, 0}^{2}\right)}}{(\sqrt{n}-\sqrt{m})^{2}+(\sqrt{n-1 / 2}+\sqrt{m-1 / 2})^{2}}\right)
$$

As can be seen, $\gamma_{u p}$ does not depend on noise power, it depends on $n$ and $m$ and $P_{f a}$ values.

\subsection{Derivation of Lower Threshold Value $\left(\gamma_{l o}\right)$}

In order to maximize the detection probability of the secondary user, $P_{m}$ should not exceed the specified value. In the proposed method, the lower threshold $\gamma_{l o}$ can be obtained based on the value of the $P_{m}$ specified by the FCC. The $P_{m}$, probability of detection is as follows:

$$
\begin{aligned}
& P_{m}=P\left(H_{1} \mid H_{0}\right)=P\left(\frac{\lambda_{\text {max }}\left(\boldsymbol{R}_{\boldsymbol{x}}\right)}{\lambda_{\text {min }}\left(\boldsymbol{R}_{\boldsymbol{x}}\right)}<\gamma_{l o}\right) \\
& P_{d}=1-\left(P\left(\lambda_{\text {max }}\left(\boldsymbol{R}_{\boldsymbol{x}}\right)>\gamma_{l o} \lambda_{\text {min }}\left(\boldsymbol{R}_{\boldsymbol{x}}\right)\right)\right)
\end{aligned}
$$

Thereby the smallest and largest eigenvalues of the $\boldsymbol{R}_{\boldsymbol{x}}$ are defined as follows:

$$
\begin{aligned}
& \lambda_{\text {max }}\left(\boldsymbol{R}_{\boldsymbol{x}}(n)\right) \approx \rho_{p}+\lambda_{\text {max }}\left(\boldsymbol{R}_{\boldsymbol{\eta}}\right) \\
& \lambda_{\text {min }}\left(\boldsymbol{R}_{\boldsymbol{x}}(n)\right) \approx \rho_{p}+\sigma_{\eta}^{2}
\end{aligned}
$$

where $\rho_{p}$ and $\rho_{l}$ indicates $\lambda_{\max }\left(\boldsymbol{R}_{\boldsymbol{x}}\right)$ and $\lambda_{\min }\left(\boldsymbol{R}_{\boldsymbol{x}}\right)$ respectively.

Then:

$$
P_{d}=1-\left(P\left(\rho_{p}+\lambda_{\max }\left(\boldsymbol{R}_{\boldsymbol{\eta}}\right)>\gamma_{l o}\left(\rho_{1}+\sigma_{\eta}^{2}\right)\right)\right)
$$

$$
\begin{aligned}
& P_{d}=1-\left[P\left(\lambda_{\max }\left(\boldsymbol{R}_{\boldsymbol{\eta}}\right) \frac{n}{\sigma_{\eta}^{2}}>\left(\gamma_{l o}\left(\rho_{1}+\sigma_{\eta}^{2}\right)-\rho_{p}\right) \frac{n}{\sigma_{\eta}^{2}}\right)\right] \\
& P_{d}=1-\left(P \left(\left(\frac{\lambda_{\max }(A(n))-\mu_{n, m, 0}}{\sigma_{c}}\right)>\right.\right. \\
& \left.\left.>\frac{\left.\left[\left(\gamma_{l o}\left(\rho_{1}+\sigma_{\eta}^{2}\right)-\rho_{p}\right) \frac{n}{\sigma_{\eta}^{2}}\right]-\mu_{n, m, 0}\right)}{\sigma_{c}}\right]\right)
\end{aligned}
$$

Thus, one side of the equation converges to the TracyWidom distribution of order 1 . If the survival function is used, $P_{d}$ is defined as:

$$
\begin{aligned}
& P_{d}=1-\left(1-\left(F\left(\frac{\left[\left(\gamma_{l o}\left(\rho_{1}+\sigma_{\eta}^{2}\right)-\rho_{p}\right) \frac{n}{\sigma_{\eta}^{2}}\right]-\mu_{n, m, 0}}{\sigma_{c}}\right)\right)\right) \\
& P_{d}=F\left(\frac{\left[\left(\gamma_{l o}\left(\rho_{1}+\sigma_{\eta}^{2}\right)-\rho_{p}\right) \frac{n}{\sigma_{\eta}^{2}}\right]-\mu_{n, m, 0}}{\sigma_{c}}\right)
\end{aligned}
$$

$$
\left(\frac{\left[\left(\gamma_{l o}\left(\rho_{1}+\sigma_{\eta}^{2}\right)-\rho_{p}\right) \frac{n}{\sigma_{\eta}^{2}}\right]-\mu_{n, m, 0}}{\sigma_{c}}\right)=F_{1}^{-1}\left(P_{d}\right)
$$

and finally. $\gamma_{l_{o}}$ is defined as follows.

$\gamma_{l o}=\frac{\left[\left(\left(-2,78 \sigma_{c}+\mu_{n, m, 0}\right) \frac{n}{\sigma_{\eta}^{2}}\right)+\rho_{p}\right]}{\rho_{1}+\sigma_{\eta}^{2}}$

From the formula, the $\gamma_{l o}$ is related to the number of samples $(n)$, number of antennas $(m)$, the maximum and minimum eigenvalues of the signal covariance matrix (including channel effect) and noise power. There are many studies in the literature to estimate the noise power. Therefore, we used the minimum mean-square error method in this study.

\section{SIMULATION}

The theoretical analyses made were simulated in the MATLAB environment. For $P_{f a}$ and $P_{m}$, the value set by the 802,22 working group was selected $\left(P_{f a}=P_{m}=0,1\right)$. BPSK modulation was applied by having selected the number of receive antennas $(m=4)$ in the secondary user. In the simulations, the MME, SEED and the proposed methods (D-SEED) are shown for Rayleigh channel 
simulation (SEED indicates $12^{\text {th }}$ reference). Algorithms for Monte Carlo simulation were run 1000 times, and ED method is shown in Fig. 1 for more accurate interpretation of the results.

In the traditional energy sensing method, noise power needs to be known. In practice, the estimated noise power may be different from the actual noise power [9, 15]. For this reason, some uncertainties are in question at the calculation of the noise power. Assuming the estimated noise power is $\tilde{\sigma}_{\eta}^{2}=\alpha \sigma_{\eta}^{2}$, the noise uncertainty factor is defined as:

$$
B=\max \left\{10 \log _{10} \alpha\right\} \mathrm{dB}
$$

It is assumed that $\alpha$ is evenly distributed in the range of $[-B, B]$. In practice, the noise uncertainty factor at the receiver can normally range from $1 \mathrm{~dB}$ to $2 \mathrm{~dB}[10,11]$. For this reason the simulations are carried out under different noise uncertainty conditions. $P_{d}$ values for different SNR values are seen in Fig. 2. It is seen that the proposed method increases the performance especially at very low SNR values and the obtained equations are theoretically proved. When looking carefully at Fig. 2, it is seen that the results of D-SEED (theoretical) and D-SEED (simulation) give closer results at high SNR values. This can be explained by Fig. 3 .

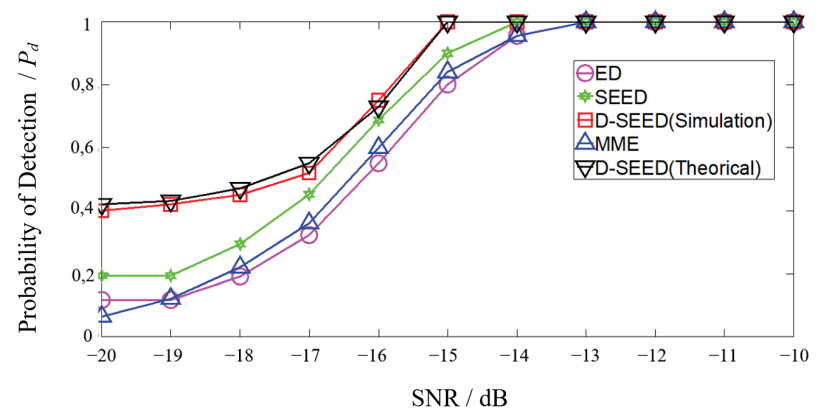

Figure 2 Rayleigh channel simulation results: $n=1000, m=4, P_{f a}=0,1, P_{d}=$ 0,1 , noise uncertainty $=0 \mathrm{~dB}$

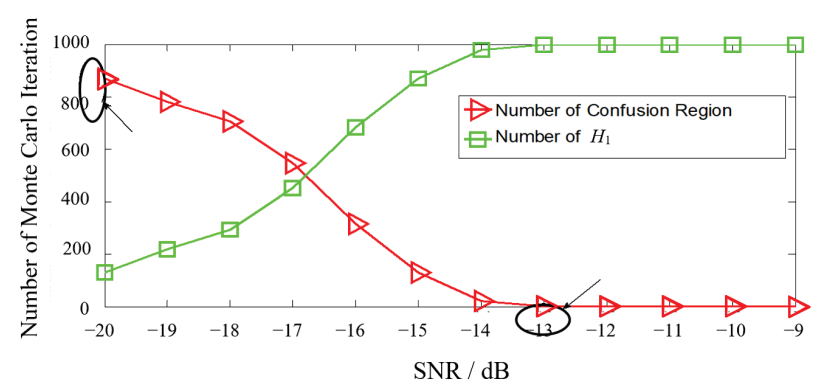

Figure 3 Number of confusion region versus SNR: noise uncertainty $=0 \mathrm{~dB}$

How many times the algorithm enters the confusion region when it is run 1000 times is given in Fig. 3. Note that the proposed method has entered the uncertainty region 830 times in the presence of $-20 \mathrm{~dB}$ noise. As well as this situation increases the probability of detection, it may have a negative effect to the time of detection. As can also be seen from the graph, the system uses the lower threshold value when the noise level is higher than $-13 \mathrm{~dB}$. However, the lower threshold value is very close and equal to the upper threshold value when the noise level is less than $-13 \mathrm{~dB}$. Therefore, it is sufficient to use only the upper threshold value for that system's correct decision on a certain noise level.

It is important that the upper threshold value is independent of noise power (Eq. (11)), while the lower threshold value is related to noise power in the method (Eq. 22). So, an accurate estimate of the noise power is required in order to calculate the lower threshold value fully. In practice, it is not possible to predict the noise power precisely because of the noise uncertainty.

Variation of threshold values for different SNR values is given in Fig. 4. The scanned area on the graph indicates the confusion region. In the graph, it appears that the system only decides by looking at the upper threshold value after a noise level of $-13 \mathrm{~dB}$.

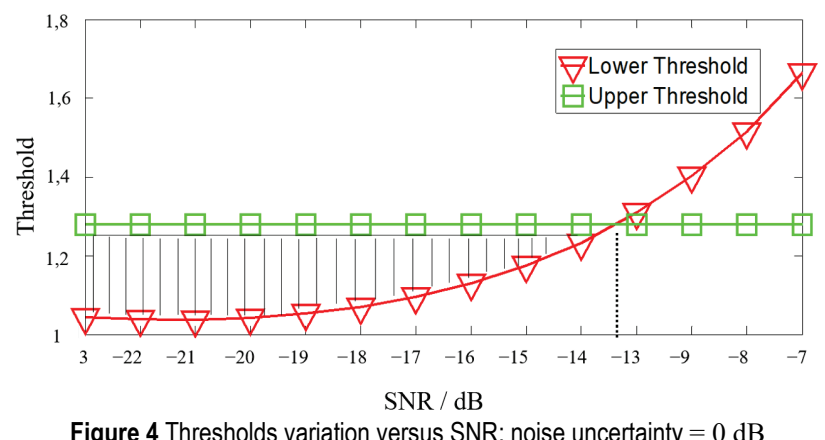

The change in SNR with $P_{d}$ for noise uncertainty of 2 $\mathrm{dB}$ is shown in Fig. 5. As can be seen from the figure, it is clear that the proposed method has better detection performance than the MME and SEED methods. Under the $2 \mathrm{~dB}$ noise uncertainty, while the detection probability of proposed method is 1 at $-12 \mathrm{~dB}$ noise, this value is $-11 \mathrm{~dB}$ for SEED. Fig. 3 and Fig. 5 are considered together, there is a 0,1 point decrease in $P_{d}$ value due to errors caused by estimating the noise power in the proposed method.

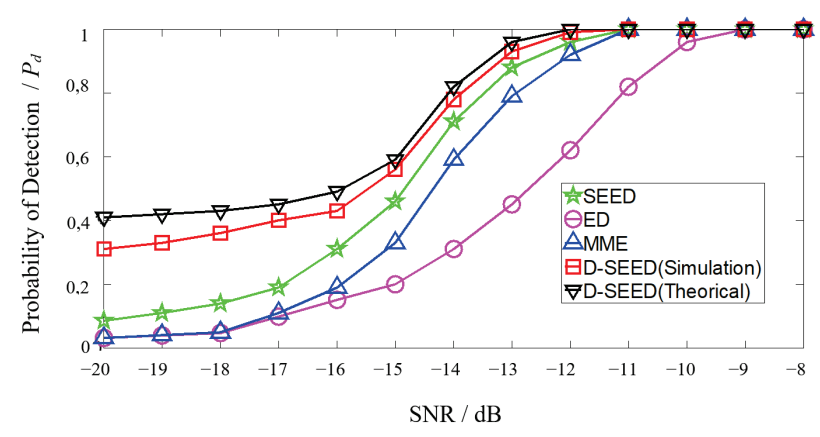

Figure 5 Rayleigh channel simulation results: $n=1000, m=4, P_{f a}=0,1, P_{d}=$ 0,1 , noise uncertainty $=2 \mathrm{~dB}$.

The method has been using the lower threshold value until $-13 \mathrm{~dB}$ and the lower threshold value depends on the noise power. Because of that, a large difference in the probability of detection is seen until $-15 \mathrm{~dB}$ level when theoretical and simulation results are compared for suggested method.

Also the detection time for the proposed dualthreshold method is directly related to the noise level. In the simulations, the second threshold usage increases the detection time by $1 \mathrm{~ms}$. Therefore, this time can be calculated by looking at Fig. 3 . 


\section{CONCLUSION}

In this study, an eigenvalue-based spectrum sensing method is proposed using a double threshold with the studentized extreme eigenvalue distribution function. The proposed method can be used where radar systems and wireless signal detection are required.

In contrast to the traditional method, a new function was used for the largest eigenvalue distribution of a random hermitian matrix. In general, dual-threshold based methods yield better results than single-threshold detection methods that use the same parameters. However, the application of energy-based and double-threshold based detection under noise uncertainty is not widely used in the literature due to unsteady states. This uncertainty problem is effectively overcome by the eigenvalue-based dualthreshold method. Especially, the dual-threshold eigenvalue based sensing, together with the proposed eigenvalue distribution function offered much better performance than traditional methods in the noise uncertainty conditions. In addition, the proposed method can be used without any prior knowledge of signal characteristic and channel.

\section{REFERENCES}

[1] Jayaweera, S. K. (2014). Signal Processing for Cognitive Radios. John Wiley \& Sons, Inc. Melbourne. https://doi.org/10.1002/9781118824818

[2] Mitola, J. \& Maguire, Jr. G. Q. (1999). Cognitive Radio: making Software Radios More. IEEE Pers. Commun., 6(4), 1318-1329. https://doi.org/10.1109/98.788210

[3] Ratnarajah, T., Vaillancourt, R., \& Alvo, M. (2005). Eigenvalues and condition numbers of complex random matrices. SIAM J. Matrix Anal.Appl., 26(2), 441-456. https://doi.org/10.1137/S089547980342204X

[4] Mishali, M. \& Eldar, Y. C. (2009). Blind Multiband Signal Reconstruction. Compressive Sensing for Analog Signals IEEE Trans. Sig. Proc., 57(3), 993-1009. https://doi.org/10.1109/TSP.2009.2012791

[5] Sobron I., Diniz, P., Martins, W., \& Velez, M. (2015). Energy detection technique for adaptive spectrum sensing. IEEE Trans. Commun., 63(3), 617-627. https://doi.org/10.1109/TCOMM.2015.2394436

[6] Chatterjee, S., Banerjee, A., Acharya, T., \& Maity, S. P. (2014). Fuzzy c-means clustering in energy detection for cooperative spectrum sensing in cognitive radio system. Proceedings of Multiple Access Communications (MACOM), 8715, 84-95 https://doi.org/10.1007/978-3-319-10262-7_8

[7] Mingchuan, Y., Yuan, L., Xiaofeng, L., \& Wenyan, T. Cyclostationary feature detection based spectrum sensing algorithm under complicated electromagnetic environment in cognitive radio networks. ChinaCommun. 12(9), 35-44. https://doi.org/10.1109/CC.2015.7275257

[8] Bhargavi, D. \& Murthy, C. (2010). Performance comparison of energy, matched-filter and cyclostationarity-based spectrum sensing. Proceedings of IEEE Eleventh International Workshop on Signal Processing Advances in Wireless Communications (SPAWC), Morocco, 1-5. https://doi.org/10.1109/SPAWC.2010.5670882

[9] Zeng, Y. \& Liang, Y. C. (2007). Covariance based signal detections for cognitive radio. Proc IEEE International Symposium on and New Frontiers in DynamicSpectrum Access Networks (DySPAN 2007) / Dublin, Ireland, 202-207. https://doi.org/10.1109/DYSPAN.2007.33
[10] Jin, M., Guo, Q., Xi, J., Youming, Li Y., Yu, Y., \& Huang, D. (2015). Spectrum Sensing Using Weighted Covariance Matrix in Rayleigh Fading Channels. IEEE Transactıons on Vehicular Technology, 64(11), 5137-5148. https://doi.org/10.1109/TVT.2014.2379924

[11] Pillay, N. \& Xu, H. J. (2011). Blind Eigenvalue-Based Spectrum Sensing for Cognitive Radio Networks. IET Comm., 6(11), 1388-1396. https://doi.org/10.1049/iet-com.2011.0506

[12] Çiflikli, C. \& Ilgin, F. Y. (2017). Covariance based spectrum sensing with studentized extreme eigenvalue. Technical gazette 25(1), 100-106. https://doi.org/10.17559/TV-20161217120341

[13] Xinzhi, Z., Feifei, G., Rong, C., \& Tao, J. (2015). Matched filter based spectrum sensing when primary user has multiple power levels. China Commun., 12(2), 21-31. https://doi.org/10.1109/CC.2015.7084399

[14] Xu, S., Shang, Y., \& Wang, H. (2009). Double thresholds based cooperative spectrum sensing against untrusted secondary users in cognitive radio networks. IEEE Vehicular Technology Conference, 519-523. https://doi.org/10.1109/VETECS.2009.5073511

[15] Charan, C. \& Pandey., R. (2016). Eigenvalue Based Double Threshold Spectrum Sensing Under Noise Uncertainty for Cognitive Radio. Optik - International Journal for Light and Electron Optics, 127(15), 5968-5975. https://doi.org/10.1016/j.ijleo.2016.04.049

[16] Shi-Qi, L., Bin-Jie, H., \& Xian-Yi, W. (2012). Hierarchical Cooperative Spectrum Sensing Based on Double Thresholds Energy Detection. IEEE Communications Letters, 16(7), 1096-1099. https://doi.org/10.1109/LCOMM.2012.050112.120765

[17] Deo, S. R. (2016). On the Tracy-Widom Approximation of Studentized Extreme Eigenvalues of Wishart Matrices. Journal of Multivariate Analysis 147, 265-278. https://doi.org/10.1016/j.jmva.2016.01.010

\section{Contact information:}

\section{Cebrail ÇíLLiKLi}

(Corresponding author)

Erciyes University, Vocational High School,

Electronic and Automation Department,

Kayseri, Turkey

cebrailc@erciyes.edu.tr

\section{Fatih Yavuz ILGIN}

Erzincan University, Vocational High School

Electronic and Automation Department

Erzincan, Turkey

E-mail: ffatihyavuzilgin@gmail.com 